\title{
Production Scheduling Oriented to Energy Consumption Optimization for Process Industry Based on Self-adaptive DE Algorithm
}

\author{
Lieping Zhang ${ }^{1,2}$, Yingxiong Luo ${ }^{3}$, Yu Zhang ${ }^{1,2}$ and Gang Song ${ }^{3}$ \\ ${ }^{1}$ Guangxi Key Laboratory of New Energy and Building Energy Saving, Guilin \\ University of Technology, Guilin, 541004, China \\ ${ }^{2}$ College of Mechanical and Control Engineering, Guilin University of \\ Technology, Guilin, 541004, China \\ ${ }^{3}$ College of Information Science and Engineering, Guilin University of \\ Technology, Guilin, 541004, China \\ zlp_gx_gl@163.com,jonhy_love@126.com, zhyuwl@163.com,zlp@glite.edu.cn
}

\begin{abstract}
Considering the production scheduling problem of processes industry and the disadvantages of conventional differential evolution algorithm, a method of production scheduling oriented to energy consumption optimization for process industry is proposed in this paper, which is based on self-adaptive differential evolution algorithm. Based on the analysis of production scheduling problems for processes industry, a production scheduling model is established, whose goal is to obtain the minimum of total process energy consumption. Since the basic differential evolution algorithm has the disadvantage that the search performance is very sensitive to the parameter settings, the self-adaptive control evolution strategy are used to control the parameters scale factor $(F)$ and crossover probability $(C R)$ to improve the global search ability and convergence speed. A practical production scheduling problem is taken as an example here, the established model and self-adaptive differential evolution algorithm are adopted to realize scheduling simulation with the minimum total process energy consumption. The simulation results show that the production scheduling method oriented to energy consumption optimization is superior to the production scheduling method oriented to process time optimization, and it can realize the goal of reducing energy consumption.
\end{abstract}

Keywords: process industry, energy consumption optimization, self-adaptive differential evolution algorithm, production scheduling

\section{Introduction}

Production scheduling is the process of realizing certain or several specific production goals by allocating limited sharing resources and collating production tasks within a given period of time. Since energy deficiency is one of the main questions confronting today's society, optimized energy consumption scheduling of production, as the predomination direction in which enterprises can further be geared on energy conservation and consumption reduction, has become one of the issues attracting intensive attention from the whole world. As an important means of realizing optimized operation of production process, production scheduling oriented to energy consumption optimization for process industry is a significant means of realizing energy conservation, improving production efficiency and economic performance, and reinforcing competitiveness [1]. As one of the hottest issues in the study of optimized scheduling theory and relevant application, production scheduling oriented to energy consumption optimization has attracted close attention from many experts and scholars at home and abroad. As discussed by Mouzona, 
Mehmet, and Yildirima [2], an energy consumption and optimization model of single machine tools was constructed, and multiple-target mathematical programming model was utilized for solutions. As discussed by Zhu, Ding, and Li [3], an energy conservationoriented multistage free forging production scheduling model was proposed and the heuristic algorithm and hungarian algorithm were used to obtain the optimal solution of energy conservation-oriented forging production scheduling. As discussed by He [4], the energy consumption characteristics of machining manufacturing system was analyzed and a modeling method of task-oriented energy consumption for machining manufacturing system was proposed. As discussed by Szijjarto, Papadokonstantakis, and Hungerbiihler [5], energy consumption in batch processes production was took into consideration, and energy usage rate and conservation potential of the path were analyzed, and these two factors were proved to have great significance to energy conservation.

An energy-efficient model for flexible flow shop scheduling was proposed to solve the makespan and the total energy consumption optimization, which was based on an improved genetic-simulated annealing algorithm [6]. A multi-objective scheduling method based on non-dominant sorting genetic algorithm was presented to reducing energy consumption as one of the objectives of job shop environment [7]. A mathematical model to minimize energy consumption costs for single machine production scheduling during production processes was proposed and genetic algorithm technology has been utilized to generate the optimal solution [8].

Differential evolution (DE) algorithm is a heuristic evolution algorithm put forward by Rainer Storn and Kenneth Price. It is a simple and effective parallel search algorithm with better robustness and rapid convergence [9]. In comparison with other evolution algorithms, DE algorithm adopts real number encoding, differential-based simple mutation operation and one-to-one strategy for competition and survival, which makes it stronger global convergence and robustness, and makes applicable to solve optimization questions in complex environment that cannot be solved by general mathematical programming methods [10]. In recent years, DE algorithm and its improved algorithm began to be adopted to solve and optimize production scheduling questions and have obtained substantial results [11-14]. Aiming at the sensitive setting of scale factor F and crossover rate $\mathrm{CR}$ in traditional $\mathrm{DE}$ algorithm and unreasonable parameter setting which will probably lead to premature convergence or stagnant algorithm, the self-adaptive control evolution strategy is proposed to make scale factor $F$ and crossover rate $\mathrm{CR}$ in dynamic change in algorithm search and optimization, with the aim to improve the optimal performance of DE algorithm. Furthermore, the proposed algorithm has been applied in production scheduling oriented to energy consumption optimization, and has obtained satisfying effect.

\section{Description of Production Scheduling Oriented to Energy Consumption Optimization for Process Industry}

\subsection{Definition of Correlated Variables}

The definition of correlated variables in terms of production scheduling oriented to energy consumption optimization for process industry is as below:

$N$ is the quantity of pre-processed products.

$P$ is the number of processes for products to be processed.

$b$ is a set of processed products, $b=\{j \mid j=1,2, L, N\}$.

$a$ is a set of manufacturing processes products, $a=\{i \mid i=1,2, L, P\}$.

$M_{j}$ is the number of devices in parallel processes of $\mathrm{j}$.

$N_{j k}$ is the number of the $\mathrm{j}$ step processing on the k device processed products. 
$S T_{i j k}$ is the start time of product $\mathrm{i}$ in equipment $\mathrm{k}$ in the $\mathrm{j}$ process of processing.

$F T_{i j k}$ is the end time of product $\mathrm{i}$ in equipment $\mathrm{k}$ in the $\mathrm{j}$ process of processing.

$E_{i j k}$ is the energy consumption of product $\mathrm{i}$ in equipment $\mathrm{k}$ in the $\mathrm{j}$ process of processing.

$E I_{j k}$ is the waiting for energy consumption of product $\mathrm{i}$ in equipment $\mathrm{k}$ in the $\mathrm{j}$ process of processing within a unit of time.

$E R O C_{j k}$ is the amount of consumed energy while initiating, preparing and shutting down the equipment $\mathrm{k}$ after processing is completed in the $\mathrm{j}$ process.

$Y_{i j k}$ is $0 / 1$ decision variable which represents if product $\mathrm{i}$ has been processed by equipment k in the j process. $Y_{i j k}=1$ represents processed, and $Y_{i j k}=0$ represents not processed.

\subsection{Mathematical Model of Production Scheduling Oriented to Energy Consumption Optimization for Process Industry}

With the purpose of facilitating modeling and algorithm solution, energy consumption considered in this paper is the amount of energy consumed from starting up to shutting down one set of equipment, namely, the total of consumed energy from the minute a machine is initiated till it is shut down after fulfilling the batch task[15].Since energy consumed by equipment is the primary source of energy consumption in production scheduling, only energy consumption in the processing of production equipment will be taken into consideration here. Other kinds of energy consumption, such as illumination, heating and ventilation will not be considered.

\subsubsection{Constraint Conditions}

The constraint conditions that the production scheduling oriented to energy consumption optimization for process industry should be meet includes the following: constraint condition in allocation, constraint condition in time, and constraint condition in sequence.

1) Constraint Condition in Allocation. Constraint condition in allocation needs to be satisfied in the processing process. In other words, any process equipment can only process one process of one product at one time, as it is shown as in equation (1).

$$
\sum_{i=1}^{M_{j}} Y_{i j k}=\left\{\begin{array}{l}
1 \\
0
\end{array}\right.
$$

In the equation, $Y_{i j k}$ is $0 / 1$ decision variable. When $Y_{i j k}=1$, it represents that equipment $\mathrm{k}$ processes product $\mathrm{i}$ in the $\mathrm{j}$ process. When $Y_{i j k}=0$, it represents that equipment $\mathrm{k}$ does not process product $\mathrm{i}$ in the $\mathrm{j}$ process. Product $i \in \beta$, process $j \in \alpha$ and equipment $k \in\left\{1,2, \cdots, M_{j}\right\}$. The total number of processes being processed in equipment $\mathrm{k}$ in each process $\mathrm{j}$ is not more than the quantity of processing equipment, namely.

$$
\sum_{k=1}^{M_{j}} N_{j k} \leq N
$$

Among which, process j satisfies $j \in \alpha$.

2) Constraint Condition in Time. As to certain processed product, constraint condition in time means the interval between the starting time and the completing 
time must be greater than or equal to the practical processing time, as it is shown as in equation (3).

$$
F T_{i j k}-S T_{i j k} \geq T_{i j k}
$$

3) Constraint Condition in Sequence. As to any processing equipment, constraint condition in sequence means before $i+1$ product is processed, the processing of $i$ product must be completed, as it is shown as in equation (4).

$$
F T_{(i+1) j k}-F T_{i j k} \geq T_{i j k}
$$

Wherein, product $i \in \beta$, process $j \in \alpha$, equipment $k \in\left\{1,2, \cdots, M_{j}\right\}, T_{i j k}$ represents the practical processing time of product $\mathrm{i}$ in equipment $\mathrm{k}$ in process $\mathrm{j}$.

\subsubsection{Mathematical Model of Energy Consumption}

The total energy consumption (TE) of the production scheduling oriented to energy consumption optimization for process industry includes the following: energy for readyopen-close (EROC), processing energy consumption (PE), and waiting for energy consumption(WE).

1) Energy for Ready-Open-Close (EROC). EROC refers to the amount of energy consumed in the preparation and follow-up work before and after product processing. For example, energy consumed in initiation, shutting down and transmission of heavy materials via cranes is included in this part. The method of calculating EROC is shown as in equation (5).

$$
E R O C=\sum_{j=1}^{P} \sum_{k=1}^{M_{j}} \text { EROC }_{j k}
$$

Among which, $j \in \alpha$ represents process, and $k \in\left\{1,2, \cdots, M_{j}\right\}$ represents equipment.

2) Processing Energy Consumption (PE). PE refers to the amount of energy needed in completing processing during industrial processing. It covers the biggest part in the total energy consumption in industrial process production scheduling. The method of calculating PE is shown as in equation (6).

$$
P E=\sum_{j=1}^{P} \sum_{k=1}^{M_{j}} \sum_{i=1}^{N_{j k}} Y_{i j k} \cdot E_{i j k}
$$

3) Waiting for Energy Consumption (WE). During the processing, it is often that there will be some idle time between the processing of two products due to constraint condition in time. Between this idle time, equipment is left unused and in idle. Waiting for energy consumption (WE) means the consumed energy in this part. The method of calculating WE is shown as in equation (7).

$$
W E=\sum_{j=1}^{P} \sum_{k=1}^{M_{j}} \sum_{i=1}^{N_{j k}} E I_{j k} \cdot\left(S T_{(i+1) j k}-F T_{i j k}\right)
$$

4) Total Energy Consumption (TE). TE in each part is the primary energy consumed in fulfilling processing tasks. The method of calculating TE is shown as in equation (8).

$$
T E=E R O C+P E+W E
$$

Mathematical model of energy consumption is a complex mathematical programming question integrating integer and nonlinearity, especially when the quantity of packages, equipment and parallel machines is large, it is extremely difficult to solve with traditional mathematical methods. 


\section{DE Algorithm Design of Production Scheduling Oriented to Energy Consumption Optimization}

\subsection{Design of Differential Encoding}

Assuming that the equipment will not be shut down once it is initiated until all the processing tasks are completed in practical production. During the processing, idling will cause unnecessary energy consumption and machine wastage. In order to reach the goal of conserving energy, reducing energy consumption and machine wastage, it is a rule in this paper that a certain product can only be processed in one set of equipment in one process, so that the number of initiated equipment can be minimized, and the rise of energy consumption in idling and machine wastage can be avoided. With the purpose of achieving an optimized scheduling plan through DE algorithm, a feasible scheduling is represented by a $\left[n^{*} \mathrm{~m}\right]$ matrix in the design of differential encoding. In the matrix, matrix row represents the sequence of tasks, and matrix line represents equipment used in the sequence. For example, matrix element A $(1,1)$ represents the equipment used in process 1 task 1 , element $\mathrm{A}(1,2)$ represents the equipment used in process 2 task 1 , element $\mathrm{A}(2$, 1) represents the equipment used in process 1 task 2 , the rest can be done in the same manner. A solution of scheduling plan can be achieved through converting the scheduling plan matrix into one-dimensional vector and representing each individual of population with this vector, and the equipment used in each process each task can be confirmed. The value range of the vector in each dimension is an integral value from 1 to the number of equipment used in the aforesaid dimension. For example, if two sets of equipment (equipment $\mathrm{A}$ and equipment $\mathrm{B}$ ) can be used in a certain process, then the value range of the individual in the dimension is [1-2], namely, the number of two sets of equipment.

\subsection{Design of Self-adaptive DE Algorithm}

The search performance and convergence rate of basic DE algorithm are sensitive to the setting of control parameters. In order to make up the deficiency caused by such sensitivity, many scholars have proposed different self-adaptive control strategies for DE algorithm. Filing DE/rand-to-pbest strategy is adopted in this paper to realize the mutation strategy in DE algorithm [16-17].

Assuming that the question to be solved includes D-dimensional variables, then the $\mathrm{i}$ individual $\mathrm{Xi}$ in population NP can be represented as below.

$$
X_{i}=\left\{x_{i}(j), x_{i}(j) \cdots, x_{i}(j)\right\}
$$

Among which, $x_{i}(j) \in\left[l_{i}, u_{j}\right], i=1,2, \cdots, N P, j=1,2, \cdots, D . x_{i}(j)$ represents the random and even initialization of real number within the range of independent variable $\left[l_{i}, u_{j}\right]$.

In each generation, the crossover rate $C R_{i}$ of each target individual $\mathrm{Xi}$ should be independently generated in accordance with the rule shown as in equation (10).

$$
\left\{\begin{array}{l}
C R_{i}=\operatorname{rndn}_{i}\left(\mu_{C R}, 0.1\right) \\
\mu_{C R}=(1-c) \times \mu_{C R}+c \times \text { mean }_{i}\left(S_{C R}\right)
\end{array}\right.
$$

When the value of $C R_{i}$ is controlled within the range of $[0,1]$, control the value within $[0,1]$ through direct interception. mean $_{i}\left(S_{C R}\right)$ is the arithmetic mean value of $C R_{i}$ gathered value of all the successfully generated individuals in generation $\mathrm{G}$. $c \in(0,1)$ is a constant.

As to each target individual, its scale factor $\mathrm{F}$ is independently generated in accordance with the rule shown as in equation (11). 


$$
\left\{\begin{array}{l}
F_{i}=r n d c_{i}\left(\mu_{F}, 0.1\right) \\
\mu_{F}=(1-c) \times \mu_{F}+c \times \text { mean }_{L}\left(S_{F}\right)
\end{array}\right.
$$

Among which, when $F_{i}>1.0$, set $F_{i}=1.0$, when $F_{i}<0$, regenerate the value. $r n d c_{i}\left(\mu_{F}, 0.1\right)$ represents Cauchy random number generated by location parameter $\mu_{F}$ and scale parameter 0.1. $S_{F}$ is the gathering of Fi value of all the successfully generated individuals in generation G. $\operatorname{mean}_{L}\left(S_{F}\right)$ is the mean value of Lehmer. Its calculation method is shown as in equation (12).

$$
\operatorname{mean}_{L}\left(S_{F}\right)=\frac{\sum_{i=1}^{\left|S_{F}\right|} F_{i}^{2}}{\sum_{i=1}^{S_{F}} F_{i}}
$$

In this paper, Griewank function and Schaffer function testing is adopted in a comparative analysis the performance of basic DE algorithm and self-adaptive DE algorithm. The results of experiment are shown as in Figure 1(a) and Figure 1(b). In the figure, the corresponding $\mathrm{x}$-coordinate represents the number of generations of arithmetic evolution, and y-coordinate represents the logarithm value of optimal individual after ten independent evolutions. It can be seen from the result of the experiment that the selfadaptive DE algorithm searches the optimal value when Griewank function is approaching the 100th generation. In comparison, basic DE algorithm searches the optimal value in around 500th generation. Likewise, in the testing with Schaffer function, the performance of the self-adaptive DE algorithm is superior to that of basic DE algorithm. This demonstrates that self-adaptive DE algorithm shows better global convergence, convergence rate, robustness and stability than basic DE algorithm.

\subsection{Fitness Function}

On the basis of the established mathematical model of energy consumption, the total energy consumption TE in production scheduling is the total of energy consumed in EROC, PE and WE, namely, fitness function. The objective of optimization in scheduling is to minimize the total energy consumption, as it is shown as in equation (13).

$$
O b j=\min (T E)
$$

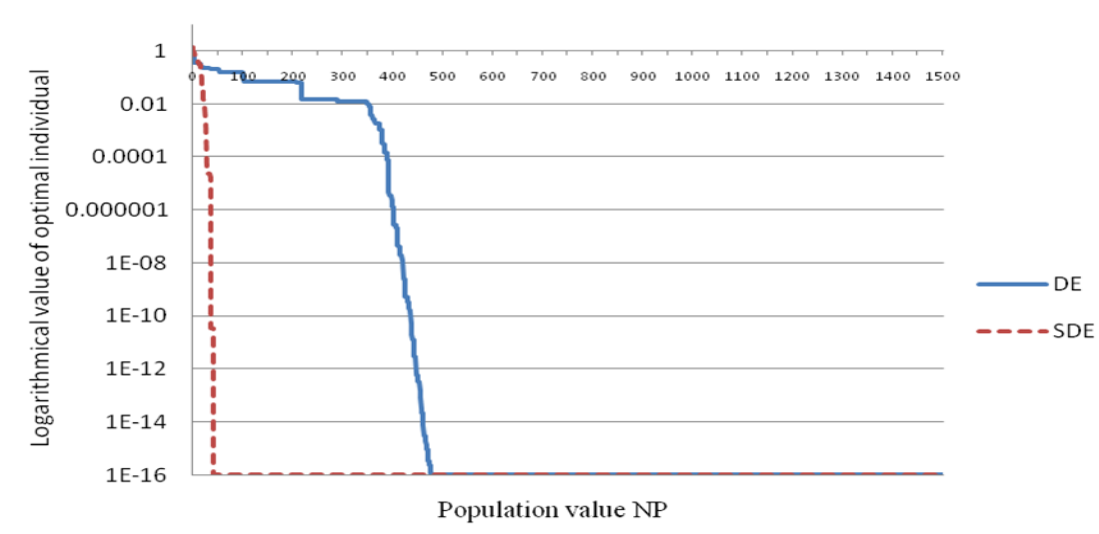

(a) Optimal value evolution of Griewank function 


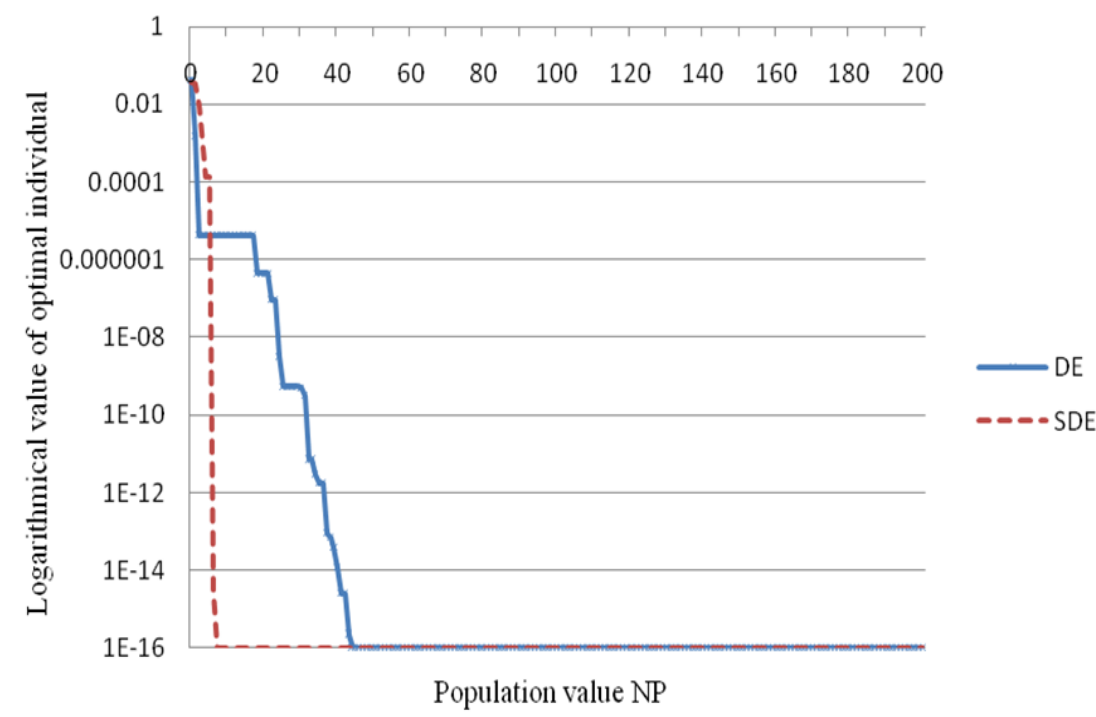

(b) Optimal value evolution of Schaffer function

\section{Figure 1. Performance Comparative Analysis between basic DE Algorithm and Self-adaptive DE Algorithm}

\subsection{Realization of Self-adaptive DE Algorithm}

The specific realization steps of self-adaptive DE algorithm of production scheduling oriented to energy consumption optimization for process industry are as below.

Step 1: initialize operation. Generate initial population PG, and each dimension of individual $X_{i}$ should be within the corresponding value range.

Step 2: calculate the fitness value of all individuals in initial population PG. In this paper, after the design of further differential encoding is completed regarding the scheduling issue, the fitness value of individuals in the population should be the amount of energy consumed in specific equipment processing represented by the individual. After individual decoding is completed, equipment number used in the processing of the individual can be obtained. After the used equipment is confirmed, calculate energy consumed in the equipment. The total consumed energy calculated via equation (13) is the fitness value.

Step 3: generate parameters crossover rate $C R$ and scale factor $F$ in self-adaption. $C R$ and $F$ corresponding to each target individual $X_{i}$ should be generated via equation (10) and equation (11).

Step 4: randomly select different individuals from the initial population PG for differential mutation, and achieve the vector $V_{i}$ of mutated individual.

Step 5: conduct mating operation on mutated individual vector $V_{i}$ and parent individual vector $X_{i}$, and generate experimental individual vector $U_{i}$.

Step 6: compare the fitness of experimental individual vector $U_{i}$ and parent individual vector $X_{i}$, preferentially pick up individuals for the next generation of population $\mathrm{PG}+1$.

Step 7: complete the judgment. If $\mathrm{G}>\mathrm{G}_{\max }$, non-inferior solutions in $\mathrm{PG}+1$ population should be regarded as output, and task scheduling is completed. Otherwise, if $\mathrm{G}=\mathrm{G}+1$, transfer to Step 3, and continue the evolution operation of G+1 generation. 


\section{Production Scheduling Simulation Based on Self-adaptive DE Algorithm}

\subsection{Description of a Production Scheduling Example}

A production enterprise is specialized in producing product 1 and product 2 . Their production process flows are shown as in Figure 2 [18]. Both the two products need two kinds of ingredients for processing. Product 1 needs ingredient 1, a solid raw material, which covers $85 \%$ of the total raw materials, and ingredient 2, a liquid raw material, which covers the rest $15 \%$. The whole production process of product 1 is constituted of five parts, blending, seasoning, cooling and filtering, etc. Likewise, Product 2 needs ingredient 1, a solid raw material, which covers $80 \%$ of the total raw materials, and ingredient 2 , a liquid raw material, which covers $20 \%$. The whole production process of product 2 includes five parts, blending, diluting, seasoning, filtering and coloring.

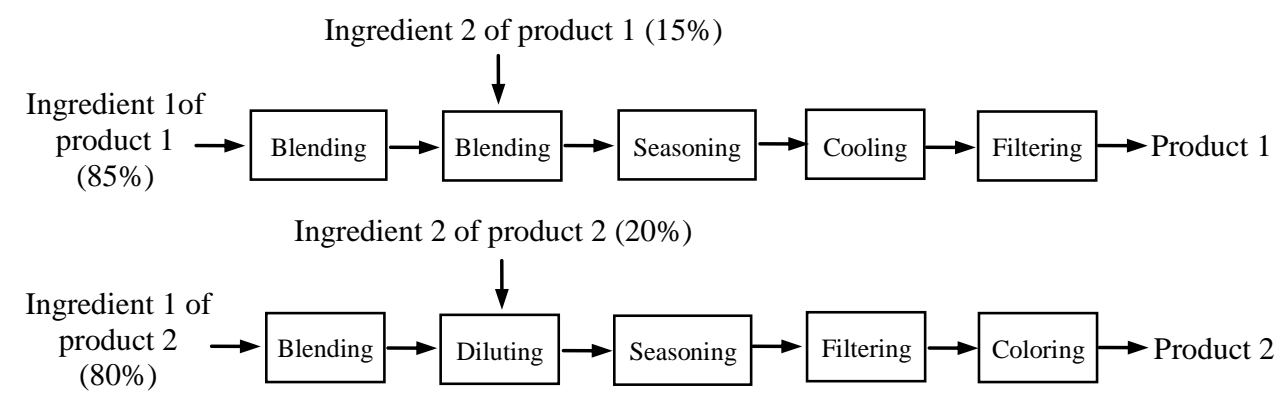

Figure 2. Schematic Diagram of Two Production Process Flows

It is assumed that the production enterprise is expected to complete the processing of two products product 1 and product 2 , four tasks in total. The corresponding tasks are shown as in Table 1 .

Table 1. Production Tasks

\begin{tabular}{ccc}
\hline production sequence & Task name & Assignments \\
1 & Product 1 & 7 \\
2 & Product 2 & 15 \\
3 & Product 2 & 18 \\
4 & Product 1 & 8 \\
\hline
\end{tabular}

The abstracted basic processing characteristics of the two products are shown as in Table 2. The number of the three premix kettles of product 1 and product 2 is $\{\mathrm{R} 11, \mathrm{R} 12$ and $R 13\}$, the number of the prepared kettle is $\{\mathrm{R} 2\}$, the number of two seasoning pots is $\{\mathrm{R} 31$ and $\mathrm{R} 32\}$, the number of the cooling kettle is $\{\mathrm{R} 4\}$, the number of the two filtering pots is $\{R 51$ and R52\}, the number of the adjustable kettle is $\{R 6\}$.

Table 2. Basic Processing Characteristics

\begin{tabular}{|c|c|c|c|c|c|c|}
\hline $\begin{array}{l}\text { Product } \\
\text { name }\end{array}$ & $\begin{array}{c}\text { Processing } \\
\text { steps }\end{array}$ & $\begin{array}{c}\text { Processing } \\
\text { requirements }\end{array}$ & $\begin{array}{l}\text { Processing } \\
\text { equipment }\end{array}$ & $\begin{array}{c}\text { Device } \\
\text { ID }\end{array}$ & $\begin{array}{c}\text { Processing } \\
\text { time }\end{array}$ & $\begin{array}{c}\text { Processing } \\
\text { capacity }\end{array}$ \\
\hline \multirow{5}{*}{$\begin{array}{l}\text { Product } \\
\text { s } 1\end{array}$} & 1 & Mix & Premixed kettle & R11 & 5 & 8 \\
\hline & 2 & Mix & Prepared kettle & $\mathrm{R} 2$ & 5 & 5 \\
\hline & 3 & Aging & Seasoning pot & R31 & 10 & 10 \\
\hline & 4 & Cooling & Cooling kettle & $\mathrm{R} 4$ & 3 & 5 \\
\hline & 5 & Filter & Filtering pot & R51 & 5 & 5 \\
\hline \multirow{5}{*}{$\begin{array}{l}\text { Product } \\
\text { s } 2\end{array}$} & 1 & Mix & Premixed kettle & $\mathrm{R} 12$ & 5 & 10 \\
\hline & 2 & Dilution & Premixed kettle & $\mathrm{R} 13$ & 5 & 5 \\
\hline & 3 & Aging & Seasoning pot & R32 & 10 & 8 \\
\hline & 4 & Filter & Filtering pot & R52 & 5 & 8 \\
\hline & 5 & Color & Adjustable kettle & R6 & 6 & 10 \\
\hline
\end{tabular}


Energy consumption data of each set of equipment in the scheduling case are shown as in Table 3. The first line represents each set of processing equipment in scheduling. 'Others' represents energy consumption in the preparation, initiation, shutting down, processing time, and idle time of each set of equipment correspondingly. The unit of the consumed energy is ton of standard coal equivalent (TCE).

Table 3. Equipment Energy Consumption

\begin{tabular}{ccccccccccc}
\hline Device name & R11 & R12 & R13 & R2 & R31 & R32 & R4 & R51 & R52 & R6 \\
EROC(TCE) & 3 & 5 & 2 & 2 & 5 & 3 & 2 & 2 & 3 & 5 \\
PE in unit time(TCE) & 10 & 13 & 8 & 8 & 13 & 10 & 8 & 8 & 10 & 13 \\
WE(TCE) & 4 & 6 & 3 & 3 & 6 & 4 & 3 & 3 & 4 & 6 \\
\hline
\end{tabular}

\subsection{Simulation Results and Discussion}

In the industrial production scheduling case, preferences of the self-adaptive DE algorithm are achieved after several experiments. The maximum cycle index $\mathrm{NC}_{\max }=200$, the number of population $\mathrm{NP}=20$, the length of individual (independent variable) $\mathrm{n}=4$. $C R$ and $F$ are generated in the evolution process under equation (9) and equation (10), and updated the value under each revolution rule. The initial location parameter is $m_{F}=0.5$ and $m_{C R}=0.5$. The corresponding scheduling Gantt diagram based on optimized energy consumption is shown as in Figure 3. In Figure 3, equipment R12 isn't initiated. The convergence graph of the total energy consumption is shown as in Figure 4.

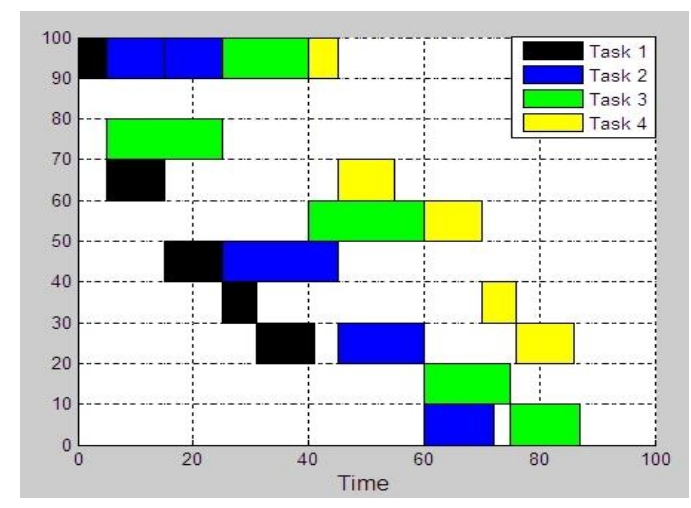

Figure 3. Scheduling Gantt Diagram based on Energy Consumption Optimization

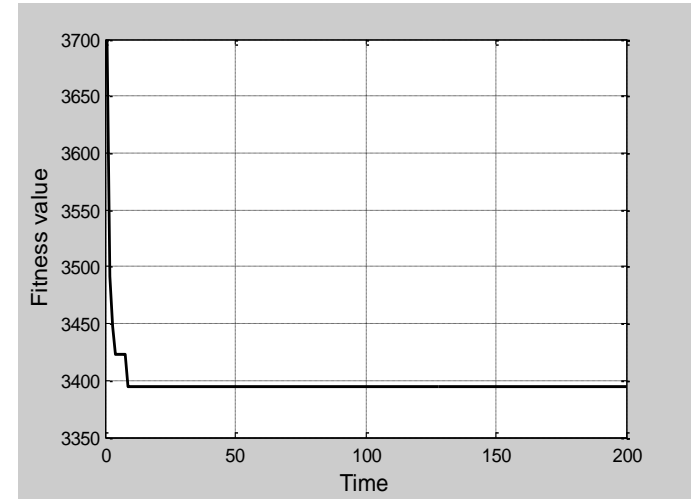

Figure 4. Convergence Curve of Total Energy Consumption based on Constrained Energy Consumption 
With the purpose of analyzing production scheduling oriented to energy consumption optimization, the scheduling result is compared with the result whose optimization aims at achieving the minimum time, as it is shown in Table 4. It can be seen in this table that with consistent parameters in population quantity and algorithm, when scheduling aims at the scheduling completion time, the total energy consumption (3656 TCE) in scheduling is higher than that aims at energy consumption optimization (3394 TCE). When scheduling aims at energy consumption optimization, the completion time is 86 unit time. When scheduling aims at the time optimization, the scheduling completion time is 77 unit time. Apparently, if scheduling aims at energy consumption optimization, although it takes a longer time in scheduling, it is worthwhile that a shorter time loss within the controllable range is used in exchange for decreasing energy consumption in scheduling.

\section{Table 4. Comparison of Energy and Time Consumption in Different} Scheduling Plans

\begin{tabular}{cccccc}
\hline Scheduling scheme & $\begin{array}{c}\text { Completion } \\
\text { time(unit time) }\end{array}$ & $\begin{array}{c}\text { TE } \\
\text { (TCE) }\end{array}$ & $\begin{array}{c}\text { PE } \\
\text { (TCE) }\end{array}$ & $\begin{array}{c}\text { EROC } \\
\text { (TCE) }\end{array}$ & $\begin{array}{c}\text { WT } \\
\text { (TCE) }\end{array}$ \\
$\begin{array}{c}\text { Time optimization } \\
\begin{array}{c}\text { Energy consumption } \\
\text { optimization }\end{array}\end{array}$ & 77 & 3656 & 2368 & 32 & 1256 \\
\hline
\end{tabular}

\section{Conclusions and Future Work}

On the basis of constructing the scheduling model oriented to energy consumption optimization for process industry and basic DE algorithm, a self-adaptive DE algorithm is designed and applied to solve production scheduling oriented to energy consumption optimization for process industry through introducing self-adaptive parameter mechanism into basic DE algorithm. The simulation results show that the designed self-adaptive DE algorithm has the advantages of shorter solution time and quicker operation, and the energy consumption optimization based on the proposed self-adaptive DE algorithm can reach the goal of energy-conserving and consumption-reducing, which offers the possibility of conserving energy and reducing consumption for enterprises in the future. However, the practical industrial production scheduling often needs a comprehensive optimization of multiple indexes. How to design a multiple-target optimized scheduling algorithm and set reasonable weight parameters in the comprehensive optimization index to seek for balance in scheduling between performance index and economic index is the direction of our further work.

\section{Acknowledgments}

This research was supported by Guangxi Natural Science Foundation (No. 2014GXNSFAA118371), and the research fund of Guangxi key laboratory of new energy and building energy saving (No.12-03-21-3).

\section{References}

[1] J. H. Xie, "Model and optimization algorithm for production scheduling based on energy saving", Master Thesis, Shanghai Jiaotong University, China, (2012).

[2] G. Mouzona, B. Mehmet and Yildirima, "Operational methodes for minimization of energy consumption of manufacuring equipment", International Journal of Production Research, no. 45, (2012), pp. 4247-4271.

[3] B. Q. Zhu, X. X. Ding and D.B. Li, "Multistage free forging production scheduling oriented to energy conservation and emission reduction", Computer Integrated manufacturing Systems, vol. 18, no. 12, (2012), pp. 2713-2722. 
[4] Y. He, B. Liu, X. D. Zhang, H. Gao and X. H. Liu, "A modeling method of task-oriented energy consumption for machining manufacturing system", Journal of Cleaner Production, no. 23, (2012), pp. 167-174.

[5] A. Szijjarto, S. Papadokonstantakis and K. Hungerbiihler, "Model-based identification and analysis of the energy saving potential in batch chemical processes", Industrial and Engineering Chemistry Research, vol. 51, no. 34, (2012), pp. 11170-11182.

[6] M. Dai, D. B. Tang, A. Giret, M. A. Salido and W. D. Li, "Energy-efficient scheduling for a flexible flow shop using an improved genetic-simulated annealing algorithm", Robotics and ComputerIntegrated Manufacturing, no. 29, (2013), pp. 418-429.

[7] Y. Liu, H. B. Dong, N. Lohse, S. Petrovic, and N. Gindy, "An investigation into minimizing total energy consumption and total weighted tardiness in job shops", Journal of Cleaner Production, no. 65, (2014), pp. 87-96.

[8] S. Fadi, O. M. Joaquin, G. S. Alvaro and O. M. Miguel, "Optimizing the production scheduling of a single machine to minimize total energy consumption costs", Journal of Cleaner Production, no. 67, (2014), pp. 197-207.

[9] R. Storn and K. Price, "Differential evolution-a simple and efficient heuristic for global optimization over continuous space", Journal of Global Optimization, vol. 11, no. 4, (1997), pp. 341-359.

[10] J. F. Yan, C. F. Guo and W. Y. Gong, "Hybrid differential evolution with convex mutation", Journal of Software, vol. 7, no. 6, (2011), pp. 2321-2328.

[11] R. Zhang, Sh. J. Song and Ch. Wu, "A hybrid differential evolution algorithm for job shop scheduling problems with expected total tardiness criterion", Applied Soft Computing, no. 13, (2013), pp. 14481458.

[12] G. L. Deng and X. Sh. Gu, "A hybrid discrete differential evolution algorithm for the no-idle permutation flow shop scheduling problem with makespan criterion", Computers \& Operations Research, no. 39, (2012), pp. 2152-2160.

[13] Ming-gang Dong and Ning Wang, "A novel hybrid differential evolution approach for large-scale zerowait scheduling with setup times of batch plants", Computers \& Chemical Engineering, vol. 45, no. 12, (2012), pp. 72-83.

[14] Q. Q. Fan, Zh. M. Lu, X. F. Yan and M. J. Guo, "Chemical process dynamic optimization based on hybrid differential evolution algorithm integrated with Alopex", Journal of Central South University of Technology (English Edition), no. 20, (2013), pp. 950-959.

[15] X. Liu, "For energy saving of process industry production scheduling model and algorithm research", Master Thesis, National university of Defense Technology, (2008).

[16] Z. H. Cai and W. Y. Gong, "Differential evolution algorithm and its application", China University of Geosciences Press, China, (2010).

[17] J. Zhang and A. C. Sanderson, "JADE: Adaptive differential evolution with optional external archive", IEEE transactions on evolutionary computation, vol. 13, no. 5, (2009), pp. 945-958.

[18] H. Zhu, "Multi-Agent based scheduling system for chemical batch production process", Master Thesis, Dalian University of Technology, (2005).

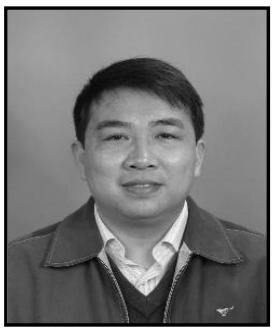

Lieping Zhang was born in 1971. He received the Ph.D degree from Kunming University of Science and Technology, Kunming, China, in 2011.He is currently a professor of college of mechanical and control engineering at Guilin University of Technology, Guangxi, China. He has published over 40 research papers in journals and international conferences. His research interests include system optimization \& scheduling and wireless sensor networks.

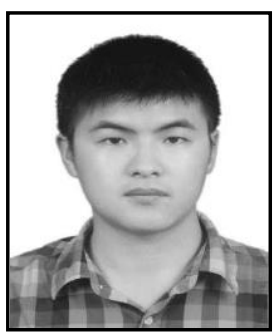

Yingxiong Luo was born in 1990. He received his B. Eng. degree in computer science from university of Jinan, Jinan, China, in 2012. He is now pursuing his master degree in college of computer science and technology, Guilin University of Technology. His research interests include production scheduling algorithm and its application. 

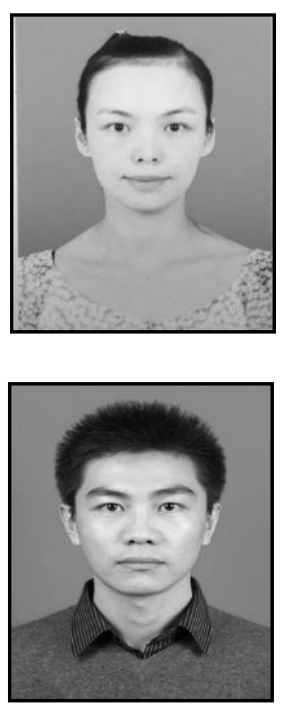

Yu Zhang was born in 1980. She received her master degree from Guilin University of Technology, Guilin, China, in 2007. She is currently an associate professor of college of mechanical and control engineering at Guilin University of Technology, Guangxi, China. She has published over 12 research papers in journals and international conferences. Her research interests include prediction of photovoltaic power generation and its application.

Gang Song was born in 1987. He received his B. Eng. degree from Bohai university, Jinzhou, China, in 2010.He is currently a postgraduate student of Guilin University of Technology, Guangxi, China. His research interests include evolution algorithm and its application. 